\title{
Ressecção via robótica de tumor pélvico obturador com componente glúteo
}

\author{
Robotic resection of a pelvic obturator tumor with gluteal component
}

Resección robótica de un tumor obturador pélvico con componente glúteo

\begin{abstract}
Henrique Acácio Barbosa ${ }^{1,2 *}$, Ana Caroline Sobrinho Dias ${ }^{1}$, Carlos Victor da Silva Nascimento ${ }^{1}$, Karen Rodrigues Quaresma Dias ${ }^{2}$, Roger Machado de Oliveira ${ }^{3}$, Leonidas Braga Dias Júnior ${ }^{1}$, Marcos de Souza Lopes Freire Filho ${ }^{2}$, Carlos Eduardo Bacchi ${ }^{4}$, Marcelo Bandeira Coelho Dias ${ }^{1}$, Patrícia Isabel Bahia Mendes Freire².
\end{abstract}

\section{RESUMO}

Objetivo: Relatar um caso de schwannoma em fossa obturadora com ressecção do componente pélvico por via robótica. Detalhamento do caso: Paciente do sexo feminino, 57 anos, dá entrada em unidade de emergência do hospital com quadro de dor em região coxofemoral direita há 12 dias, evoluindo com febre e piora do quadro álgico. Os exames de imagem mostraram tumoração ocluindo a fossa obturadora direita e com extensão via forâmen obturatório ao compartimento interglúteo direito. Optado por abordagem combinada via robótica para acesso pélvico e posterior convencional para o compartimento glúteo. No procedimento cirúrgico, o acesso via robótica para a pelve possibilitou diminuição do dano aos nervos pélvicos e baixo volume de perda sanguínea. $O$ estudo anatomopatológico evidenciou neoplasia formada pela proliferação de células fusiformes, dispostas em feixes multidirecionais, com a formação de corpúsculos de Verocay e células histiocitoides, necessitando de análise imunohistoquímica para melhor elucidação diagnóstica. Essa, por sua vez, revelou expressão difusa para proteína S-100, confirmando a hipótese de schwannoma. Considerações Finais: Dessa forma, a cirurgia robótica tende a crescer cada vez mais como alternativa na abordagem de tumores pélvicos de difícil acesso, visando a alcançar uma melhor recuperação pós-operatória e aumento da qualidade de vida do paciente.

Palavras-chave: Cirurgia oncológica, Laparoscopia, Neoplasias, Patologia.

\begin{abstract}
Objective: To report a case of schwannoma in a filling pit with resection of the pelvic component by robotic approach. Case details: A 57-year-old female patient was admitted to the emergency unit of the hospital with pain in the right hip region for 12 days, progressing with fever and worsening of the pain. Imaging exams showed a tumor that occluded the right obturator fossa and extended via obturator foramen to the right intergluteal compartment. Opted for a combined robotic approach for pelvic and posterior conventional access to the gluteal compartment. In the surgical procedure, robotic access to the pelvis enabled decreased damage to the pelvic nerves and low volume of blood loss. The anatomopathological study showed a neoplasm formed by the proliferation of spindle cells, arranged in multidirectional bundles, with the formation of Verocay bodies and histiocytoid cells, requiring immunohistochemical analysis for better diagnostic clarification. This, in turn, revealed diffuse expression for protein S-100, confirming the schwannoma hypothesis. Final Considerations: In this way, robotic surgery tends to grow more and more as an alternative to approach pelvic tumors that are difficult to access, aiming to achieve a better postoperative recovery and increase the patient's quality of life.
\end{abstract}

Key words: Surgical oncology, Robot-assisted surgery, Neoplasms, Pathology.

1Universidade do Estado do Pará (UEPA), Belém - Pará. *E-mail: henriqueacacio@gmail.com

${ }^{2}$ Hospital Porto Dias, Belém - Pará.

${ }^{3}$ Laboratório Bacchi Consultoria em Patologia, Botucatu - São Paulo.

SUBMETIDO EM: 2/2020

ACEITO EM: 3/2020

PUBLICADO EM: 4/2020

REAS/EJCH | Vol.12(7) | e3150 | DOI: https://doi.org/10.25248/reas.e3150.2020 Página 1 de 8 


\section{RESUMEN}

Objetivo: Informar un caso de schwannoma en un pozo de llenado con resección del componente pélvico mediante abordaje robótico. Detalles del caso: Una paciente de 57 años ingresó en la unidad de emergencia del hospital con dolor en la región de la cadera derecha durante 12 días, que progresó con fiebre y empeoró el dolor. Los exámenes de imágenes mostraron un tumor que ocluía la fosa obturadora derecha y se extendía a través del agujero obturador hasta el compartimento interglúteo derecho. Optó por un enfoque robótico combinado para el acceso convencional pélvico y posterior al compartimento glúteo. En el procedimiento quirúrgico, el acceso robótico a la pelvis permitió un menor daño a los nervios pélvicos y un bajo volumen de pérdida de sangre. El estudio anatomopatológico mostró una neoplasia formada por la proliferación de células fusiformes, dispuestas en haces multidireccionales, con la formación de cuerpos de Verocay y células histiocitoides, que requieren un análisis inmunohistoquímico para una mejor clarificación diagnóstica. Esto, a su vez, reveló una expresión difusa para la proteína S-100, confirmando la hipótesis del schwannoma. Consideraciones finales: De esta manera, la cirugía robótica tiende a crecer cada vez más como una alternativa para abordar los tumores pélvicos de difícil acceso, con el objetivo de lograr una mejor recuperación postoperatoria y aumentar la calidad de vida del paciente.

Palabras clave: Cirugía de cáncer, Cirugía robótica, Neoplasia, Patología.

\section{INTRODUÇÃO}

Os schwannomas, ou neuromas, correspondem a neoplasias benignas que se originam a partir da proliferação desordenada das células de Schwann, responsáveis pela formação da bainha de mielina nos nervos periféricos (AGNIHOTRI S, et al., 2016). A principal etiologia desse tipo de tumor está relacionada à deficiência de proteínas supressoras de tumor nas células de Schwann, devido à perda de heterozigosidade do gene associado à neurofibromatose tipo 2 (NF2), podendo ocorrer em até $60 \%$ desse tipo de tumor (AGNIHOTRI S, et al., 2016; CHAVES JPG, et al., 2019).

Essa neoplasia corresponde à principal forma de tumor benigno em nervos periféricos, com maior incidência em pacientes entre 20 e 70 anos, e acometendo ambos os sexos de maneira semelhante. Os principais sítios de acometimento dessas neoformações são a região cefálica (45\%) e membros inferiores (32.6\%), sendo o acometimento retroperitoneal representado por apenas $0.7 \%$ dos casos (CHAVES JPG, et al., 2019; CARVALHO BJ, et al., 2019).

A apresentação clínica dos schwannomas retroperitoneais é variável, podendo ser assintomática, com o diagnóstico ocorrendo de maneira acidental em até $34 \%$ dos casos, ou apresentando sintomatologia decorrente da compressão de estruturas adjacentes, em cerca de $50 \%$ dos casos (LI Q, et al., 2007). Estas, por sua vez, são representadas pela dor e distensão abdominal, mas podem a chegar a casos extremos, com a aderência a estruturas nobres, levando a hidronefrose e compressão de bifurcação aórtica (RAGURAJAPRAKASH K, et al., 2019).

O diagnóstico deve ser feito inicialmente com a ressonância magnética, a qual demonstra uma massa bem definida hipo ou isodensa em T1 e hiperdensa em T2, podendo apresentar degeneração cística e expansão do forame da raiz nervosa, com destruição óssea, hemorragias subagudas e calcificações (CHAN TS et al., 2019; SHEN Y et al., 2018).

A punção guiada por ultrassonografia pode auxiliar na elucidação diagnóstica inicial, tendo em vista que a caracterização do schwannoma apenas por exames de imagem pode levar a diagnósticos equivocados. Esta, em geral, demonstra agregação de células fibrilares e alongadas, as quais sugerem a possibilidade do neuroma, porém não são capazes de assegurar o diagnóstico (ZHANG L, et al., 2018). Contudo, a confirmação diagnóstica e, principalmente, a exclusão de malignidade, só podem ser realizadas pelas análises histopatológica e imunohistoquímica, as quais só são possíveis após a extração completa do tumor por via cirúrgica (MEYER A e BILLINGS SD, 2020).

Classicamente, a abordagem de ressecção por via aberta é tida como preferencial para a remoção de tumores pélvicos, especialmente em se tratando de tumores de maiores dimensões, com a via laparoscópica e robótica sendo reservada para lesões menores (DI FURIA M, et al., 2018). Em revisão realizada por 
Colecchia L, et al. (2020), foi verificada que a principal abordagem desse tipo de neoplasia é a por via aberta, com abordagem anterior sendo considerada mais adequada. Além disso, a ressecção fragmentada também foi vista como superior à remoção em bloco nos tumores pélvicos, pelo risco de lesão da inervação sacral, podendo levar à sequelas no pós-operatório dos pacientes (COLECCHIA L, et al., 2020).

No presente estudo, relatamos um caso de schwannoma em fossa obturadora com ressecção do componente pélvico por via robótica.

\section{DETALHAMENTO DO CASO}

Paciente do sexo feminino, 57 anos, dá entrada em unidade de emergência do hospital com quadro de dor em região coxofemoral direita há 12 dias, evoluindo com febre e piora do quadro álgico. Internou em hospital do interior do estado, onde apresentou quadro de leucocitose, além de ter realizado tomografia computadorizada de bacia mostrando volumosa formação expansiva com densidade de partes moles e pequeno foco denso na sua periferia, de formato lobulado, localizada na fossa obturatória e entre a musculatura do compartimento posterior da coxa, protruindo através do forame obturatório, medindo cerca de $9,6 \mathrm{~cm}$, de origem indeterminada.

Durante o período de internação no interior do estado, realizou 3 esquemas de antibioticoterapia, iniciando com Ceftriaxona, seguido pela associação de Cefepime com Levofloxacino e, por fim, Imipenem, para tratamento de infecção do trato urinário, sem melhora do quadro. Foi encaminhada ao presente centro para investigação de causas neoplásicas (febre de origem obscura). Como antecedentes pessoais, apresentava hérnia de disco intervertebral. Ainda em serviço externo realizou ressonância magnética de pelve, onde evidenciou-se tumoração de grande volume ocluindo a fossa obturadora direita e com extensão via forâmen obturatório ao compartimento interglúteo direito (Figura 1).

Figura 1 - RM de pelve com visualização de tumor em fossa obturatória, com conteúdo pélvico (seta vermelha) e glúteo (seta azul).

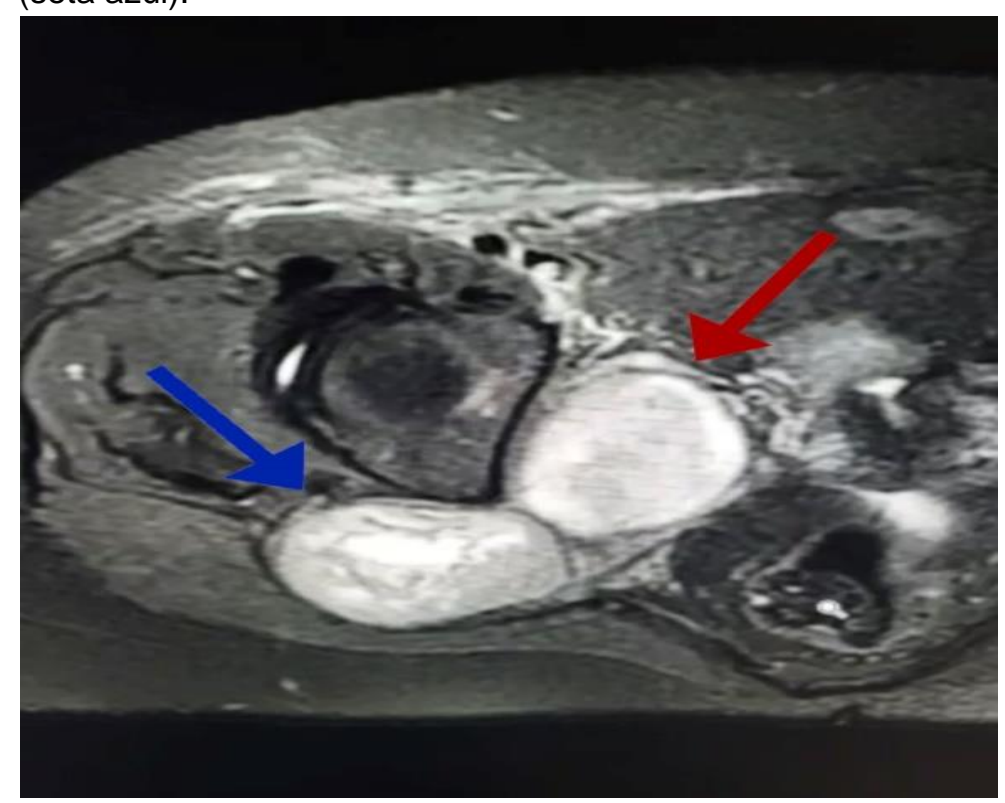

Fonte: Barbosa HA, et al., 2020.

$\mathrm{Na}$ chegada ao presente centro, de posse dos exames radiológicos em mídia física, foi solicitada de imediato a avaliação da Radiologia Intervencionista para a realização de biópsia transcutânea guiada por ultrassonografia, após a qual a paciente evoluiu com piora importante da lombociatalgia, sendo prescrita com moduladores para dor neuropática (Pregabalina, Morfina e Duloxetina). 
O estudo anatomopatológico coletado na biópsia transcutânea revelou fragmentos de neoplasia fusocelular, com ausência de anaplasia ou necrose, sendo então levantada a hipótese de schwannoma. Após melhora do quadro clínico, paciente iniciou a realização de exames pré-operatórios.

No procedimento cirúrgico, foi realizado docking pélvico modificado ao invés do docking pélvico clássico, tendo como órgão alvo a fossa obturadora direita, melhorando assim a factibilidade via robótica de todo o tempo pélvico.

Desta forma, foram contempladas a abordagem profunda abaixo do nervo obturatório e identificação de todas as raízes sacrais, findando a abordagem anterior com a dissecção da massa já na emergência do forâmen em comunicação com espaço interglúteo.

Houve necessidade de linfadenectomia pélvica tática, uma vez que a massa competia com o espaço pélvico, assim como a identificação do ureter direito e nervo obturador direito. O tempo de docking foi de 2 minutos, com o tempo de console finalizado em 1 hora e 55 minutos (Figura 2).

Figura 2 - Tumor visualizado (seta azul) pela abordagem robótica e relação com inervação pélvica, representada pelo nervo obturador (seta vermelha).

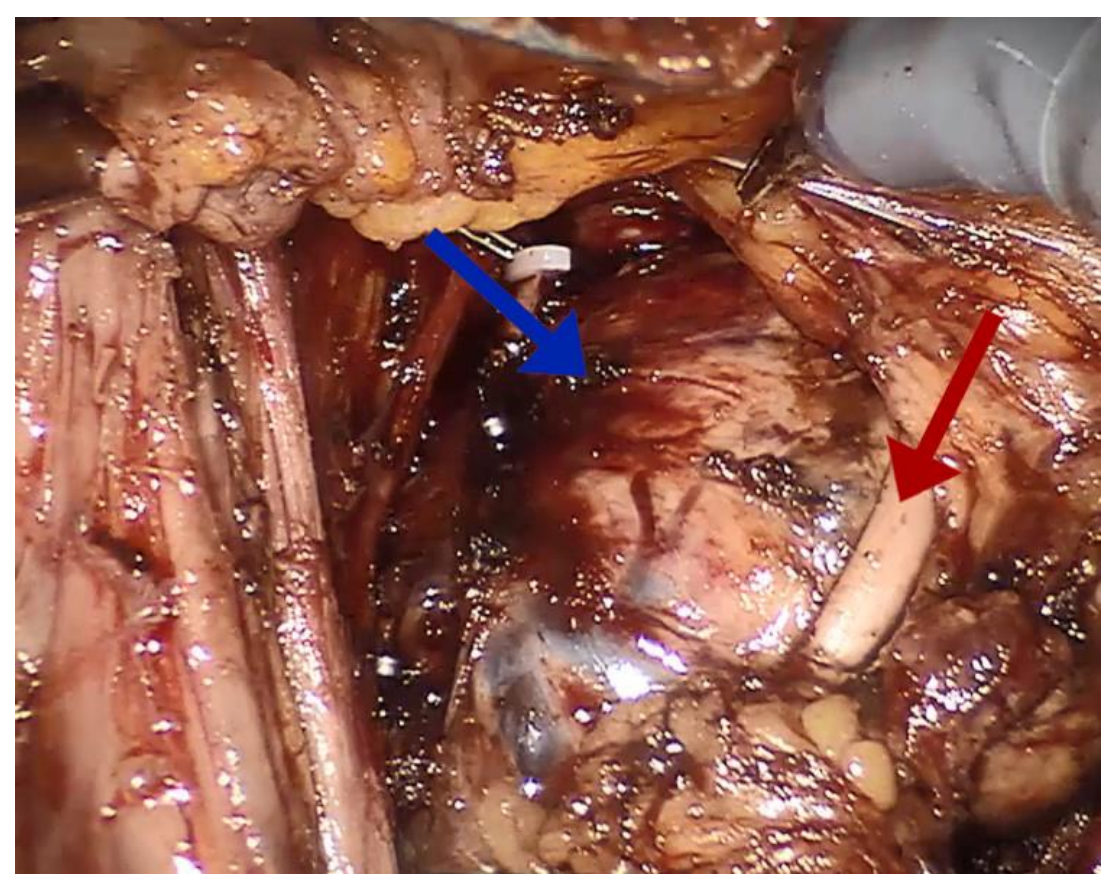

Fonte: Barbosa HA, et al., 2020.

Concluído o tempo robótico, foi realizado uma incisão auxiliar de Gibson para extração da peça e drenagem da fossa a fim de evitar linfocele. Para a remoção da outra porção do tumor, foi optado por uma abordagem aberta da região glútea direita para a retirada da peça. Ao fim do procedimento, foram removidos um tumor pélvico direito, um linfonodo retroperitoneal e um tumor glúteo direito, os quais foram enviadas para estudo anatomopatológico.

A análise histopatológica do tumor glúteo direito evidenciou neoplasia formada pela proliferação de células fusiformes, dispostas em feixes multidirecionais, com a formação de corpúsculos de Verocay. Foram notadas em meio à lesão células histiocitoides, com núcleos redondos, sem atipias e com citoplasmas claros, sendo sugerida a necessidade de análise imunohistoquímica para melhor elucidação diagnóstica. Essa, por sua vez, revelou expressão difusa para proteína S-100, confirmando a hipótese de schwannoma (Figura 3, Figura 4 e Figura 5). 
Após um dia de internação em leito de Unidade de Terapia Intensiva no pós-operatório imediato, paciente seguiu para leito de Enfermaria, apresentando boa evolução clínica. Teve como intercorrência Delirium. Recebeu alta hospitalar, em bom estado geral, no $7^{\circ}$ dia de pós-operatório.

Figura 3 - Detalhe mostrando células fusiformes formando corpúsculos de Verocay (Hematoxilina-Eosina, aumento original de 40x).

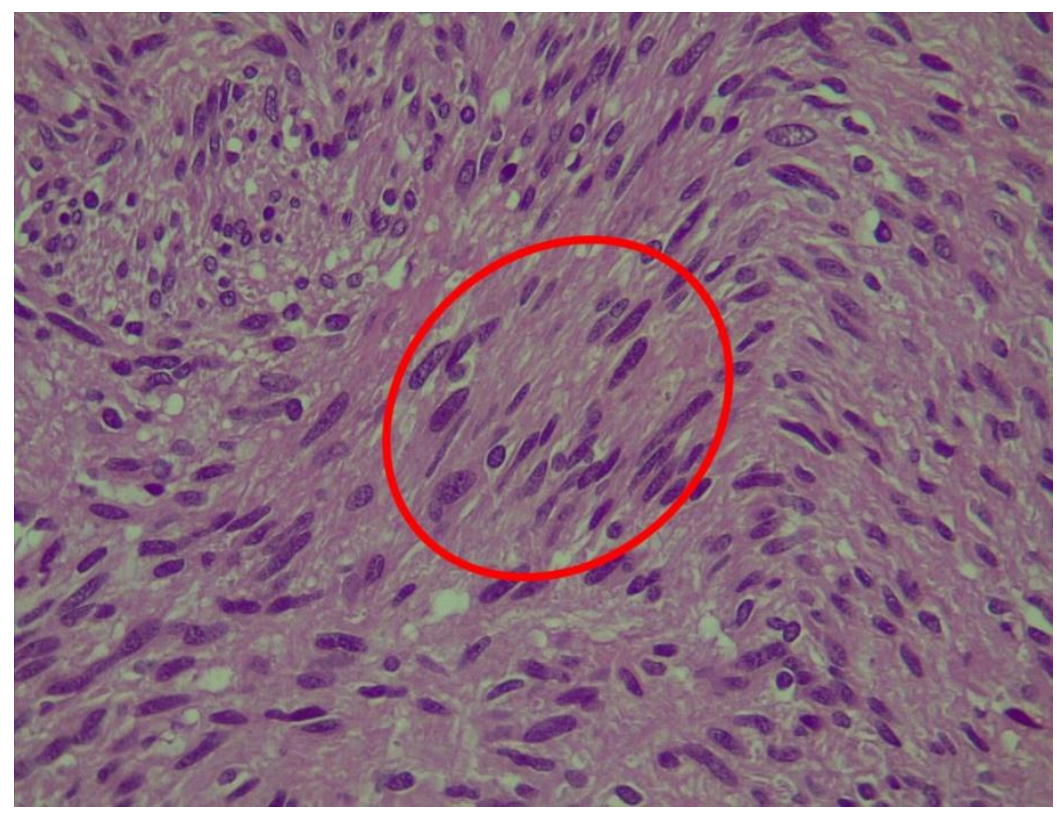

Fonte: Barbosa HA, et al., 2020.

Figura 4 - Detalhe mostrando células com núcleos redondos, sem atipias e com citoplasmas claros (Hematoxilina-Eosina, aumento original de $20 x$ ).

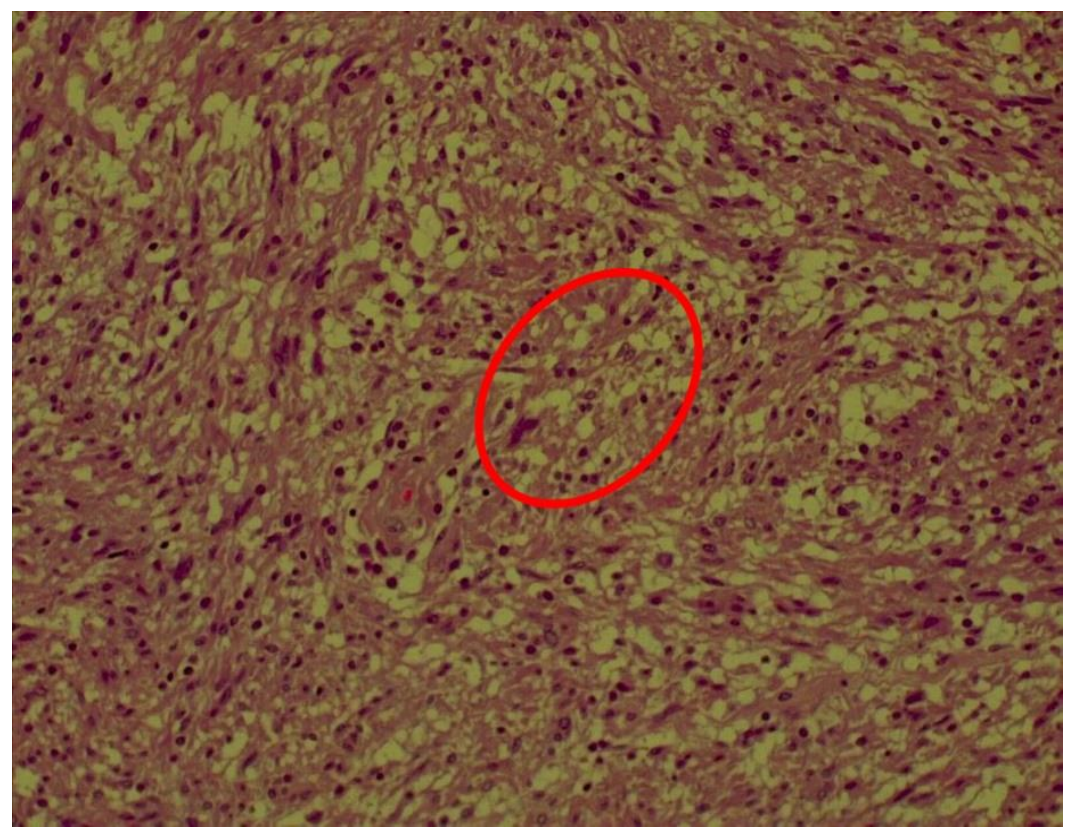

Fonte: Barbosa HA, et al., 2020. 
Figura 5 - Tumor Glúteo direito, coloração demonstrando expressão para proteína S-100

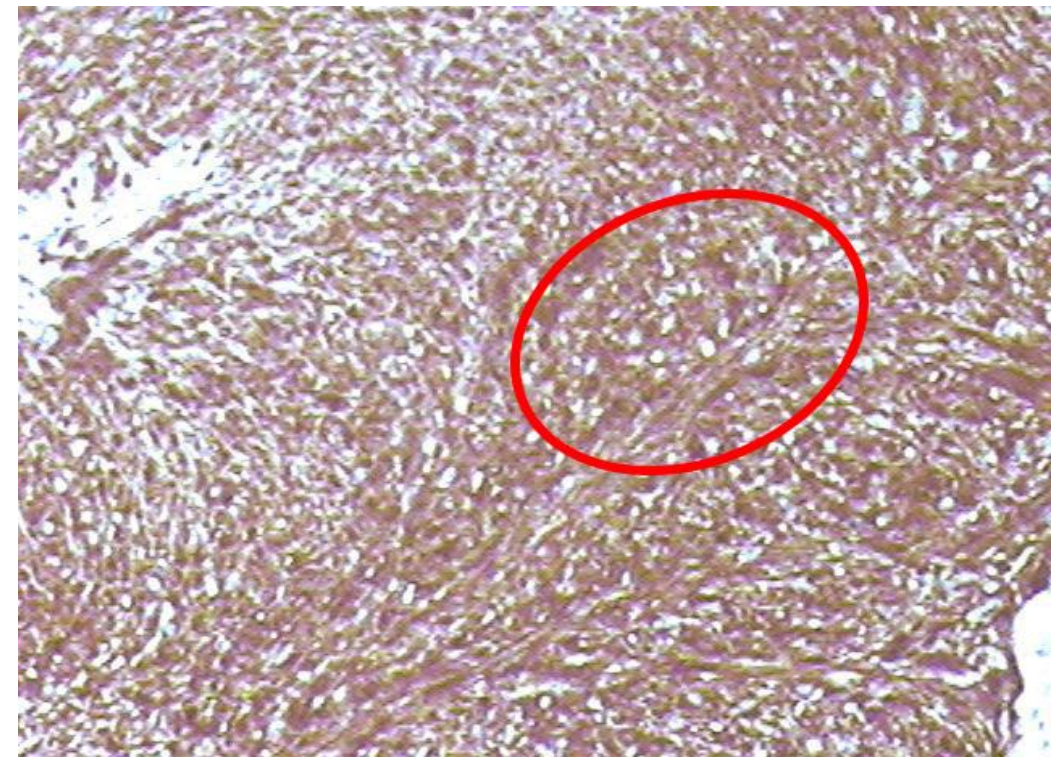

Fonte: Barbosa HA, et al., 2020.

\section{DISCUSSÃO}

A correta excisão dos schwannomas retroperitoneais representa um desafio na prática cirúrgica, uma vez que a correta retirada do tumor está diretamente relacionada à possibilidade de recidivas, bem como a elucidação diagnóstica, pela análise histopatológica. Devido à elevada elasticidade do espaço retroperitoneal, é comum que o paciente não apresente quaisquer sintomatologias nas fases iniciais do tumor, levando a um diagnóstico e terapêutica tardios, gerando tumores de maiores dimensões, e com maior dificuldade na remoção destes (HOLBROOK C, SALEEM N, 2017).

Inicialmente, a cirurgia radical aberta era vista como a principal opção terapêutica, visando a facilitar o acesso ao espaço retroperitoneal e reduzir o risco de lesão a estruturas adjacentes, como o pâncreas, os rins e vasos de grande calibre. Além disso, essa técnica era preferencial na excisão de neuroma de grande volume $(>3 \mathrm{~cm})$, bem como para a remoção de linfonodos circundantes, com o objetivo de diminuir as recidivas (KURIAKOSE S, et al., 2014).

Com a evolução tecnológica, a videolaparoscopia foi idealizada como técnica ideal para a abordagem cirúrgica nessa localização. O estudo realizado por Ji JH, et al. (2017) demonstrou que os pacientes submetidos à retirada por via laparoscópica apresentaram um menor período de internação no pós-operatório, se comparados aos pacientes submetidos à cirurgia aberta. Contudo, a realização de procedimentos mais delicados, em especial naqueles em que há íntima relação do schwannoma com estruturas nobres como a veia cava inferior e as veias ilíacas, exige um elevado grau técnico tanto da equipe da cirurgia geral, quanto da cirurgia vascular (MACCIÒ A, et al., 2019).

Nos últimos anos, a cirurgia robótica vem ganhando cada vez mais força na intervenção cirúrgica de tumores retroperitoneais, sendo considerada uma opção promissora para o futuro. O aumento da destreza, o filtro para o tremor natural das mãos humanas e a visualização em três dimensões são as principais vantagens da cirurgia robótica em relação à cirurgia laparoscópica convencional (KAHRAMANGIL B, BERBER E, 2018). A maior precisão da técnica robótica é especialmente valiosa em tumores de dimensões elevadas $(>5 \mathrm{~cm})$ ou nos quais há aderência a estruturas adjacentes. Nesses grupos, a cirurgia robótica está relacionada a uma perda de sangue estimada em $50 \mathrm{~mL}$, um tempo médio de estadia hospitalar no pós-operatório de 4.8 dias, morbidades presentes em apenas $5 \%$ dos pacientes em um acompanhamento após 90 dias, além de redução na dor no pós-operatório imediato, ao comparar-se com a laparoscopia. (KAHRAMANGIL B, BERBER E, 2018; LIU Q, et al., 2018) 
$\mathrm{Na}$ abordagem de schwannomas retroperitoneais, em especial, a cirurgia robótica é uma excelente opção terapêutica, tendo em vista que a apresentação de tais neoplasias no fossa obturatória, como a observada na paciente do presente estudo e no relatado por Chopra $S$ et al. (2018), podem levar à lesão do nervo obturador. Nesse caso, os pacientes podem evoluir com rotação externa do quadril, adução e perda da sensibilidade no aspecto medial da coxa, sendo necessária a utilização de uma técnica operatória mais refinada, como a cirurgia robótica (CHOPRA S, et al., 2018)

À observação intra-operatória, os neuromas apresentam-se macroscopicamente como tumores isolados, bem delimitados e circundados por uma cápsula fibrosa, sendo a excisão completa da neoplasia sem a rotura da cápsula uma medida fundamental para evitar-se recidivas (RAGU R., et al., 2017). Após a remoção, a peça deve ser enviada para a análise histopatológica e imunohistoquímica para a confirmação diagnóstica, bem como para a diferenciação entre as formas benignas e malignas de schwannoma (BAIRD B, et al., 2020).

O estudo anatomopatológico dos schwannomas benignos são, em geral, circundados por uma cápsula ou pseudocápsula, bem como uma apresentação bifásica composta por um componente celular (Antoni A) e um componente mixoide (Antoni B). A porção celular do tumor possui os característicos corpúsculos de Verocay, com núcleos em paliçada circundando processos fibrilares, enquanto o componente mixoide demonstra vasos aumentados, com espaços irregulares entre si, além de paredes espessadas e hialinizadas (KOCHARYAN $\mathrm{AH}$, et al., 2020). As apresentações malignas dos neuromas são raras e apresentam uma configuração distinta dos benignos, tendo como principais características histológicas a intensa atividade mitótica e as atipias celulares (MEYER A e BILLINGS SD, 2020).

A avaliação imunohistoquímica é fundamental na caracterização específica das linhagens histiocitoides das células presentes no componente mixoide do tumor. Todas as variações dos schwannomas são difusas e fortemente positivas para o marcador $\mathrm{S} 100$ e SOX10, sendo também positivo o marcador GFAP (proteína acidificante fibrilar glial) e citoqueratinas nos neuromas localizados em mediastino e abdome (MEYER A e BILLINGS SD, 2020).

No presente caso, foi verificada a presença do schwannoma na região retroperitonial, a qual é infrequente na literatura, representando apenas $0.7 \%$ dos casos desse tipo de neoplasia (CHAVES JPG, et al., 2019). Devido à localização atípica, o a investigação diagnóstica foi realizada por diversas abordagens, como a ressonância nuclear magnética como forma de rastreio bem como a biópsia guiada por ultrassonografia com o objetivo de identificar a natureza da tumoração, conforme o recomendado na literatura médica (CHAN TS, et al., 2019; SHEN Y, et al., 2018).

Contudo, a exclusão de malignidade e a confirmação diagnóstica deve ser realizada pela extração completa do tumor, a qual foi realizada por via robótica, que está relacionada à menor perda volêmica, menor período de internação pós-operatório e redução na dor pós-procedimento (KAHRAMANGIL B, BERBER E, 2018; LIU Q, et al., 2018). Tais vantagens foram vistas como essenciais na paciente deste relato, pelo histórico de dor neuropática e infecções do trato urinário de repetição.

Porém, os achados clínicos e condutas tomadas no presente caso não devem ser generalizadas para todos os pacientes com schwannomas em localização retroperitoneal, por se tratar apenas de um relato de caso. Dessa forma, novos estudos clínicos randomizados e de coorte devem ser realizados, visando a avaliar qual abordagem é a mais adequada nesses pacientes e como será o prognóstico desses pacientes a longo prazo, respectivamente. Ademais, populações mais amplas devem ser analisadas, para que uma amostra estatística significante seja considerada e, assim, seja possível a elaboração de consensos de investigação e terapêutica para tais pacientes.

Os schwannomas correspondem à principal forma de tumor benigno em nervos periféricos, com maior incidência em pacientes entre 20 e 70 anos, e acometendo ambos os sexos de maneira semelhante. A localização retroperitoneal, em especial na fossa obturadora, é rara, porém pode evoluir com diversas complicações, tendo em vista seu desenvolvimento insidioso. Devido ao difícil acesso e a proximidade à estruturas nobres, a abordagem por cirurgia robótica vem ganhando cada vez mais força na remoção cirúrgica de tumores pélvicos, como o schwannoma. Essa técnica permite um aumento na destreza, possibilita maior 
chance de preservação nervosa, redução na perda sanguínea, e permite ao médico analisar de maneira tridimensional o sítio cirúrgico. Além disso, o auxílio do robô está relacionado a um menor período de internação e diminuição no quadro álgico do pós-operatório imediato. Dessa forma, a cirurgia robótica tende a crescer cada vez mais como alternativa na abordagem de tumores pélvicos de difícil acesso, visando a alcançar uma melhor recuperação pós-operatória e aumento da qualidade de vida do paciente.

\section{REFERÊNCIAS}

1. AGNIHOTRI S, et al. The genomic landscape of schwannoma. Nat Genet 2016; 48(11): 1339-48.

2. BAIRD B, et al. Perineal schwannoma: A case report with novel genitourinary association and histopathology. Urol Case Reports 2020; 28: 101021. Doi: 10.1016/j.eucr.2019.101021.

3. CARVALHO BJ, et al. Laparoscopically excised retroperitoneal presacral Schwannoma: atypical pre and postoperative manifestations - case report. BMC Surg 2019; 19(1): 1-5. Doi: 10.1186/s12893-019-0611-8.

4. CHAN TS, et al. Primary pelvic retroperitoneal ancient schwannoma-a rare diagnosis of pelvic complex cystic lesion. Hong Kong Med J 2019; 25(2): 160.e1-160.e3. Doi: 10.12809/hkmj187188.

5. CHAVES JPG, et al. Giant Schwannoma of the Median Nerve: A Case Report and Literature Review. Asian J Oncol 2019; 5(1): 36-8. Doi: 10.11138/ccmbm/2017.14.2.265.

6. CHOPRA S, et al. Robot-Assisted Laparoscopic Resection of a Pelvic Schwannoma. Urol Case Reports 2017; 11 : 63-5. Doi: 10.1016/j.eucr.2016.11.027.

7. COLECCHIA L, et al. Giant Pelvic Schwannoma : Case Report and Review of the Literature. Dig Dis Sci 2020. Doi: 10.1007/s10620-020-06128-2.

8. DI FURIA M, et al. Advantage of lapa-roscopic resection for pelvic schwannoma: Case report and review of the literature. Int J Surg Case Rep. 2018;45:38-41

9. HOLBROOK C, SALEEM N. Retroperitoneal schwannoma: an unusual cause of abdominal distention. BMJ Case Rep, 2017.

10. $\mathrm{JI} \mathrm{JH}$, et al. Laparoscopic resection of retroperitoneal benign neurilemmoma. Ann Surg Treat Res 2017;92:149-55

11. KAHRAMANGIL B, BERBER E. Comparison of posterior retroperitoneal and transabdominal lateral approaches in robotic adrenalectomy: an analysis of 200 cases. Surg Endosc 2018; 32(4): 1984-9. Doi: 10.1007/s00464-017-58941.

12. KOCHARYAN AH, et al. Atypical Schwannoma: A 10-year experience. Am J Otolaryngol - Head Neck Med Surg 2020; 41(1). Doi: 10.1016/j.amjoto.2019.102309.

13. KURIAKOSE S, et al. Unique surgical issues in the management of a giant retroperitoneal schwannoma and brief review of literature. Case Rep Med 2014.

14. LI Q, et al. Analysis of 82 cases of retroperitoneal schwannoma. ANZ J Surg 2007; 77(4): 237-40. Doi: 10.1111/j.1445-2197.2007.04025.x.

15. LIU Q, et al. Robotic resection of benign nonadrenal retroperitoneal tumors: A consecutive case series. Int $\mathrm{J}$ Surg 2018; 55: 188-92. Doi: 10.1016/j.ijsu.2018.04.013.

16. MACCIÒ A, et al. An interdisciplinary approach for laparoscopic removal of a large retroperitoneal pelvic schwannoma attached to vital vessels: A case report. Med (United States) 2019; 98(51). Doi: 10.1097/MD.0000000000018149.

17. MEYER A, BILLINGS SD. What's new in nerve sheath tumors. Virchows Arch 2020; 476(1): 65-80. Doi: 10.1007/s00428-019-02671-0.

18. RAGU R, et al. Robotic excision of large retroperitoneal Schwannoma (with video). J Visc Surg 2017; 154(4): $297-9$. Doi: 10.1016/j.jviscsurg.2017.05.003.

19. RAGURAJAPRAKASH K, et al. Giant Invasive Sacral Schwannoma with Aortic Bifurcation Compression and Hydronephrosis. World Neurosurg 2019. Doi: 10.1016/j.wneu.2019.12.088.

20. SHEN Y, et al. MR imaging features of benign retroperitoneal paragangliomas and schwannomas. BMC Neurol 2018; 18(1): 1-8. Doi: 10.1186/s12883-017-0998-8.

21. ZHANG L, et al. Surgical management of retroperitoneal schwannoma complicated with severe hydronephrosis: $A$ case report. Med (United States) 2018; 97(39). Doi: 10.1097/MD.0000000000012528. 